\title{
AIAA 2002-0072 \\ POD Analysis of Sound Generation by a Turbulent Jet
}

J. B. Freund

University of Illinois at Urbana-Champaign, Urbana, IL 61801

T. Colonius

California Institute of Technology, Pasadena, CA 91125

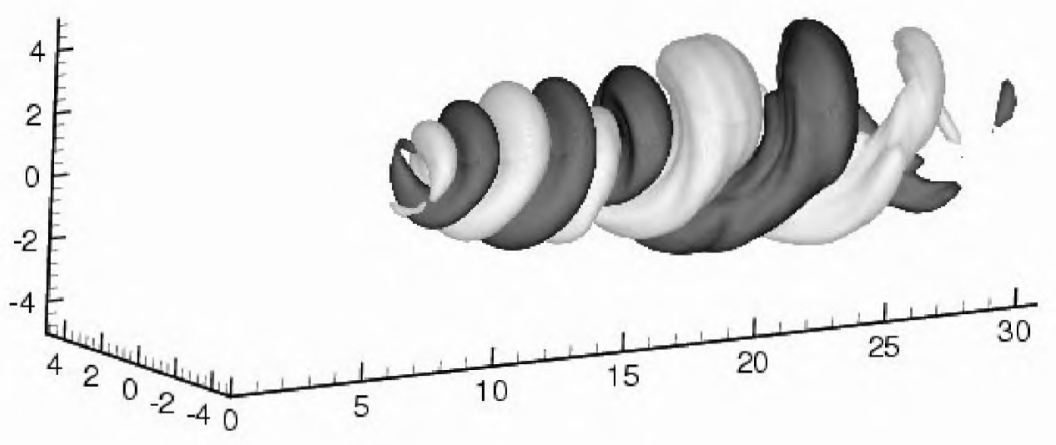

\section{0th AIAA Aerospace Sciences Meeting and Exhibit January 14-17, 2002/Reno, NV}




\title{
POD Analysis of Sound Generation by a Turbulent Jet
}

\author{
J. B. Freund* \\ University of Illinois at Urbana-Champaign, Urbana, IL 61801 \\ T. Colonius ${ }^{\dagger}$ \\ California Institute of Technology, Pasadena, CA 91125
}

\begin{abstract}
A Proper Orthogonal Decomposition (POD) is constructed for a Mach 0.9 turbulent jet using a well-validated direct numerical simulation database. Norms are defined based on near-field volume integrals of pressure, turbulence kinetic energy, streamwise velocity, and total enthalpy, two-dimensional integrals of streamswise velocity (to match experimental measurements), and far-field integrals of pressure over a sphere. We find substantially different POD modes for the different norms, and their efficiency at representing the full data is strongly dependent upon the norm and specifically which data we attempt to represent. To reproduce near-field turbulence statistics requires relatively few modes computed by a kinetic energy or pressure norm. However, a large number of the POD modes computed using a near-field norm are required to represent the sound field. The dominant near-field POD modes computed with either the near-field pressure norm or the sound field norm have the structure of wave packets.
\end{abstract}

\section{Nomenclature}

Sound speed

$J \quad$ Number of POD modes retained

Re Reynolds number

$M \quad$ Mach number

$N \quad$ Total number of snapshots

$n$

$r$

$r_{o}$

$t$

$v_{x}$

$v_{r}$

$v_{\theta}$

$x$

$\psi$

$\rho$

$\varphi$

$\theta$

\section{Subscripts \\ j Jet exit \\ $\infty \quad$ Ambient}

Other

() Base flow (potentially time dependent)

' Acoustic perturbation

() Inner product

*Assistant Professor, Theoretical and Applied Mechanics. AIAA member.

${ }^{\dagger}$ Associate Professor, Division of Engineering and Applied Science. AIAA member.

Copyright (c) 2002 by J. B. Freund. Published by the American Institute of Aeronautics and Astronautics. Inc. with permission.

\section{Introduction}

The Proper Orthogonal Decomposition (POD) ${ }^{1}$ uses data to generate a set of basis functions that optimally represent the flow's energy as defined by a userselected norm. This basis is optimal in the sense that a finite number of these orthogonal modes represent more of the flow energy than any other set of orthogonal modes. For this reason, the POD is often used to define energetic structures in the flow, ${ }^{2-6}$ which can be used in modeling either by the inspiration they provide for phenomenological models or quantitatively by Galerkin projection of a small number of modes onto the governing equations to generate a reduced-order model.

The POD has recently been used to study jets..$^{2,3,7}$ Norms involving pressure fluctuation just outside the jet give modes that look like a wave packets, ${ }^{2}$ similar to what one would expect for a growing and decaying instability wave. Citriniti \& George ${ }^{7}$ obtained POD modes using a streamwise velocity norm at a single $x$ locations and found that aside from the axisymmetric component, the most energetic modes had azimuthal Fourier coefficient $n=5$ in the shear-layer portion of the jet. Further downstream, this same group found $n=2$ modes to dominate. ${ }^{8}$

In past, it has not been possible to measure or compute three-dimensional POD modes and, in turn, quantify their dynamical significance. However, a recent simulation discussed in the following section provides the necessary database for such a study.

The mere existence of POD modes does not guarantee that they have dynamical significance, especially to a particular process such as sound generation. Indeed the sound radiated by turbulence is a very small frac- 
tion of the total energy. In other words, norms that highlight the near-field energetic structures may not be an efficient way to represent the noise generating flow, even if the dynamics of these structures are ultimately responsible for generating the sound. An intriguing question is whether an appropriate norm can be defined that would efficiently represent the sound producing dynamics of the flow, and a long term goal of the present work is to address this issue. In other words, we seek to determine what is a good norm to define such that relatively few POD modes contribute to the generation of radiated sound. This, we hope, will in turn lead to phenomenological models for sound radiation by large-scale structures in turbulent jets.

The immediate goals of the present paper are more modest: we examine the structure of three dimensional POD modes in the turbulent jet and compare those that are produced using different norms. These including some two-dimensional norms related to previous experimental measurements, and norms defined over the acoustic field to highlight the sound generation process. We also study the efficiency with which the POD modes are able to reconstruct various pointwise statistics, such as turbulence kinetic energy or sound pressure level.

\section{Simulation Database}

The simulation database is reported on in detail by Freund. ${ }^{9}$ It is a Mach 0.9, Reynolds number 3600 jet with uniform stagnation temperature. Results agree with the mean flow development, sound field directivity, and noise spectrum of the corresponding experiments of Stromberg et al. ${ }^{10}$ In addition, downstream of the potential core, where the turbulence becomes fully developed, Reynolds stresses match those measured in much higher-Reynolds-number jets. ${ }^{9,}, 11,12$ Though the Reynolds number is low, kinetic energy spectra are broad-banded, two-point velocity correlations decay rapidly in space, and physically resolved dissipation of turbulence kinetic energy is a significant factor in the overall energy budget.

In the course of the simulation, all flow variables were saved every 20 numerical time steps of $\Delta t=$ $0.0085 r_{o} / a_{\infty}$ on every other mesh point in space. This resolution is sufficient to compute most flow quantities. There are, in all, 2333 such file saves which are used to compute POD modes in this study.

\section{The Proper Orthogonal Decomposition}

In this section we briefly outline the notation and properties of the POD and the procedures we used to compute it. Results are stated here without proof. Our approach follows closely that of Rowley; ${ }^{13}$ see that text for further details and references.

We seek a representation for a vector of flow quantities, functions of space and time $\mathbf{q}(x, t)$, as an expansion in vector-valued orthogonal modes, $\varphi_{j}(x)$. The
POD expansion provides an optimally convergent series representation of a specified $L_{2}$ norm of $\mathbf{q}$. For incompressible flow fields, this norm is typically taken to be the fluctuation kinetic energy. In general. we can specify $\mathbf{q}$, the region over which the norm is defined. and how the individual components of $\mathbf{q}$ are weighted in the norm.

For compressible flows, the best choices of variables, norms, and weightings are not obvious, as many of the (interrelated) dependent variables, including thermodynamic quantities. can be important. Rowley ${ }^{13}$ found that the stagnation enthalpy was particularly useful, which in our cylindrical coordinates would give $\mathbf{q}=\left(v_{x}, v_{r}, v_{\theta}, a\right)$, where $a$ the speed of sound, and the norm

$$
\|\mathbf{q}\|^{2}=\int_{\Omega} \frac{2 a^{2}}{\gamma-1}+v_{x}^{2}+v_{r}^{2}+v_{\theta}^{2} d V,
$$

where again, $\Omega$ is the region of interest. In the present study, we are primarily interested in acoustics, and in order to compare with earlier studies, ${ }^{2}$ we would like the pressure of the POD modes to be defined. So we generalize and use $\mathbf{q}=\left(v_{x}, v_{r}, v_{\theta}, a, p\right)$ with scaling factor $\boldsymbol{\alpha}=\left(\alpha_{1}, \ldots, \alpha_{5}\right)$ and define

$$
\|\mathbf{q}\|^{2}=\int_{\Omega} \alpha_{1} v_{x}^{2}+\alpha_{2} v_{r}^{2}+\alpha_{3} v_{\theta}^{2}+\alpha_{4} a^{2}+\alpha_{5} p^{2} d V,
$$

where a consistent non-dimensionalization of $\mathbf{q}$ is inplied. The constants $\boldsymbol{\alpha}$ determine the specific norm. Choosing $\boldsymbol{\alpha}=\left(1,1,1, \frac{2}{\gamma-1}, 0\right)$ recovers the stagnation enthalpy norm used by Rowley, ${ }^{13}$ and $\boldsymbol{\alpha}=(1,1,1,0,0)$ recovers the standard kinetic energy norm often used in incompressible flow.

It is well known that for homogeneous (periodic) coordinate directions, Fourier modes are identical to POD modes. We anticipate this result by starting with the azimuthal Fourier transform of $\mathbf{q}$.

$$
\mathbf{q}(x, r, \theta, t)=\overline{\mathbf{q}}(x, r)+\sum_{m=-N_{H} / 2}^{N_{\theta} / 2} \mathbf{q}^{m}(x, r, t) e^{i m \theta},
$$

where we have removed the nean, $\overline{\mathbf{q}}$, and computed POD modes for each $m$ as

$$
\mathbf{q}^{m}(x, r, t)=\sum_{j=1}^{N} a_{j}^{m}(t) \varphi_{j}^{m}(x, r) \quad m=0, \text { ldots }, M_{\theta}
$$

To reduce computation we take $M_{\theta}=9$ and use the $\mathbf{q}^{-m}=\mathbf{q}^{m *}$ symmetry.

\section{Method of snapshots}

For clarity, we drop the superscript $m$ in this section. In the method of snapshots, ${ }^{14}$ we use the simulation data saved at the $N=2333$ different times denoted by $t_{j}$. The POD modes are

$$
\varphi_{j}(x, r)=\sum_{i=1}^{N} c_{i}^{(j)} \mathbf{q}\left(x, r, t_{i}\right)
$$


where each $c_{j}^{(k)}, k=1,2, \ldots, N$, is an eigenvector of

$$
\sum_{j=1}^{N} M_{i j} c_{j}^{(k)}=\lambda^{(k)} c_{i}^{(k)},
$$

and the matrix $M_{i j}$ is the inner product of the snapshots

$$
M_{i j}=\left\langle\mathbf{q}\left(x, r, t_{j}\right), \mathbf{q}\left(x, r, t_{i}\right)\right\rangle,
$$

where the inner product of $\mathbf{q}_{1}$ and $\mathbf{q}_{2}$ is

$$
\begin{aligned}
\left\langle\mathbf{q}_{1}, \mathbf{q}_{2}\right\rangle=\int_{\Omega} \alpha_{1} v_{x_{1}} v_{x_{2}} & +\alpha_{2} v_{r_{1}} v_{r_{2}}+\alpha_{3} v_{\theta_{1}} v_{\theta_{2}} \\
& +\alpha_{4} a_{1} a_{2}+\alpha_{5} p_{1} p_{2} d V .
\end{aligned}
$$

Thus the defining $L_{2}$ norm is (2).

\section{Norms considered}

We have computed vector valued POD modes, $\varphi_{j}$ using the norms listed in table 1 . The first 3 are integrations over the computational domain, with $\boldsymbol{\alpha}$ chosen so that the kernel represents the fluctuating kinetic energy, pressure, and streamwise velocity, respectively. The fourth norm uses only the streamwise velocity, and integrates over a slice at a single streamwise positions, $x=18 r_{o}$. This is chosen to match the experimental setup of George and co-workers. ${ }^{6-8,15}$ Finally, we consider a norm defined by the integration of the sound pressure level over a portion of a sphere at $60 r_{o}$ from the origin, and extending to spherical angles between 10 and 90 degrees (limited by the truncation of the extended acoustic domain discussed below).

\section{The Near Field}

\section{Energy representation}

The relative eigenvalues for the modes defined with the $p-3 \mathrm{~d}$ and $K-3 \mathrm{~d}$ norms (see table 1 ) are shown in figure 1. The nine largest eigenvalues are shown for each of the first 10 azimuthal modes numbers. The most energetic $K$-3d mode is at $n=2$, while the most energetic $p$-3d mode is at $n=1$. The $p$-3d mode energies decay more rapidly with both $n$ and eigenvalue number at a particular $n$. Azimuthal spectra have, in the past, shown that the $p$ Fourier coefficients peaks at smaller $n$ than the $u$ Fourier coefficients. ${ }^{16}$ At small $n$, the energetic $p$ - $3 \mathrm{~d}$ modes come roughly in pairs that seem to represent propagation with nearly constant phase speed. In table 2, the percentage of energy captured in $J$ modes, for varying $J$, is listed for all the norms discussed in this paper.

Using the $u-2 \mathrm{~d}$ norm (see table 1), Citriniti \& George $^{7}$ found that in the shear layer region of a turbulent jet, the most energetic POD mode had $n=0$. The next most energetic mode was at $n=4$, and their second most energetic mode at each azimuthal mode number typically contained less than one-third the energy of the most energetic mode at the same $n$. It would be misleading to make a direct comparison to this data
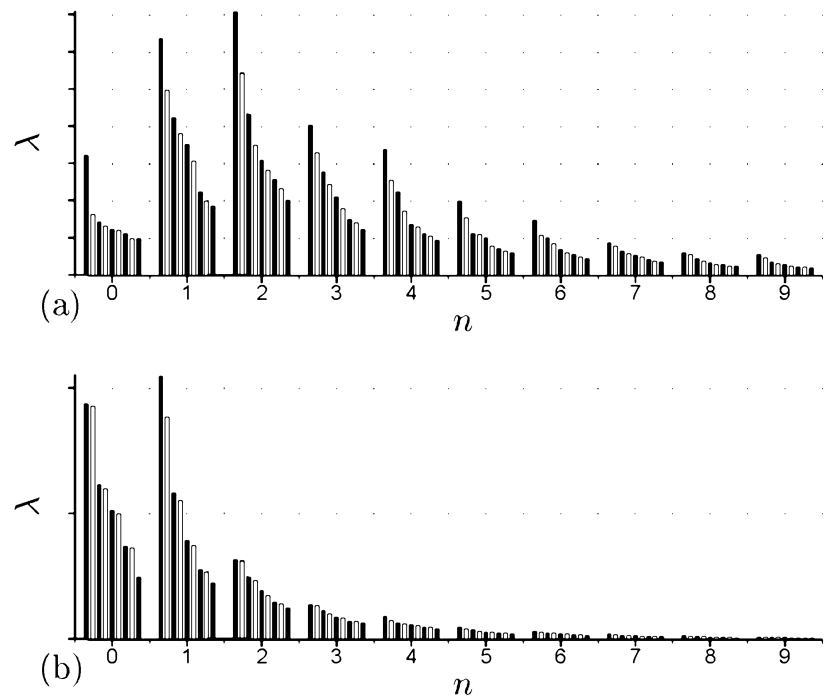

Fig. 1 Relative mode energies: (a) threedimensional $K-3 d$, and (b) $p-3 d$ as defined in (table 1). The nine largest eigenvalues for each azimuthal mode are shown.

\begin{tabular}{cccccc}
\hline$J$ & $p-3 \mathrm{~d}$ & $K-3 \mathrm{~d}$ & $U-3 \mathrm{~d}$ & $U-2 \mathrm{~d}$ & $p$-sp \\
\hline 1 & 4.0 & 2.2 & 3.1 & 4.7 & 27 \\
5 & 15 & 8.6 & 11 & 20 & 56 \\
10 & 25 & 14 & 18 & 35 & 70 \\
50 & 53 & 36 & 44 & 72 & 96 \\
100 & 64 & 49 & 57 & 85 & 99 \\
500 & 88 & 81 & 84 & 99 & 100 \\
\hline
\end{tabular}

Table 2 Percent induced norm captured with $J$ POD modes.

because our low-Reynolds-number jet is laminar for much of the shear layer region. It is only downstreanı that it becomes turbulent, starting to match Reynolds stresses of high-Reynolds-number jets downstream of the potential core's closing. However, George and his group have more recently obtained POD modes further downstream in the jet. ${ }^{6,8,15}$ In figure 2 (a) the relative energies of the modes computed using the $u-2 \mathrm{~d}$ norm at $x=18 r_{o}$ in the same way as these references. ${ }^{6,8,15}$ Like Jung et al. ${ }^{8}$ saw at $x=12 r_{o},{ }^{*}$ we too see that the most energetic mode is at $n=2$ and energies fall off for both higher and lower $n$ 's. In agreement with Jung, ${ }^{6}$ the next most energetic mode at each is $n$ is approximately one-third the energy at the most energetic mode.

However, the $u$-3d norm shown in figure 2 (b) reflects the greater complexity of the full threedimensional flow as compared to a two-dimensional slice. Although the peak energy is still at $n=2$ and energy of the most energetic modes at neighboring $n$ 's drop in a similar fashion as in two dimensions, the

*A direct comparison at the same downstream location does not seem possible because our potential core is longer since the turbulence is slower to develop in this low-Reynolds-number jet. 


\begin{tabular}{ccccc}
\hline Norm \# & Name & $\Omega$ & $d V$ & $\boldsymbol{\alpha}$ \\
\hline 1 & $K$-3d & $x \in(1,33), r \in(0,8)$ & $2 \pi r d r d x$ & $(1,1,1,0,0)$ \\
2 & $p$-3d & $x \in(1,33), r \in(0,8)$ & $2 \pi r d r d x$ & $(0,0,0,0,1)$ \\
3 & $U$-3d & $x \in(1,33), r \in(0,8)$ & $2 \pi r d r d x$ & $(1,0,0,0,0)$ \\
4 & $U$-2d & $x=18 r_{o}, r \in(0,8)$ & $2 \pi r d r$ & $(1,0,0,0,0)$ \\
5 & $p$-sp & $\sqrt{x^{2}+r^{2}}=R=60 r_{o}$. & $4 \pi R^{2} \sin \psi d \psi$ & $(0,0,0,0,1)$ \\
& & $\psi\left(10^{\circ}, 90^{\circ}\right)$ & & \\
\hline
\end{tabular}

Table 1 Various norms (and regions of integration) considered.
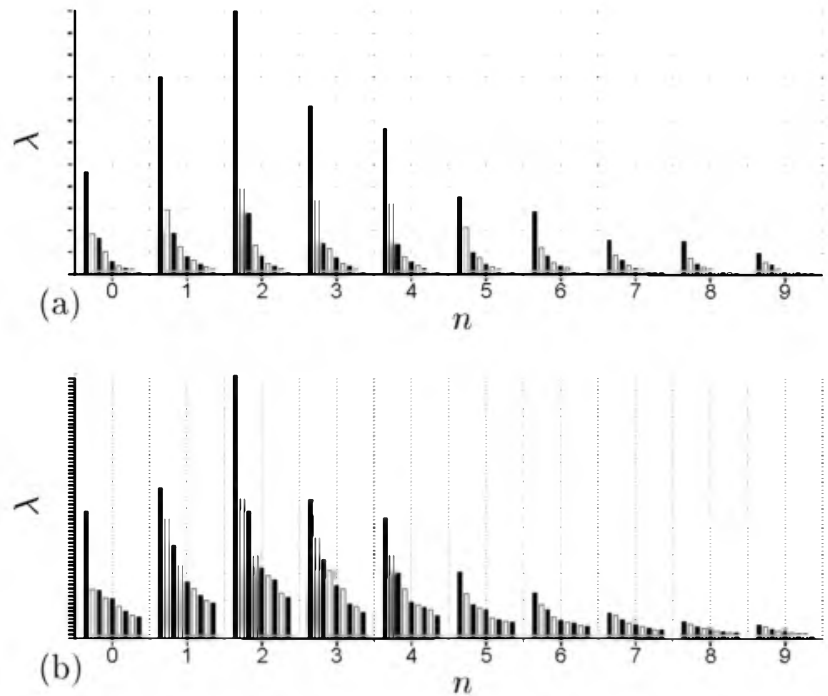

Fig. 2 Relative mode energies for streamwise velocity norms defined in table 1: (a) $u$-2d norm; (b) $u$-3d norm. The nine largest eigenvalues for each azimuthal mode are shown.

drop off in energy at a fixed $n$ is significantly slower. The most energetic modes now account for much less of the total energy of the flow.

\section{POD Modes}

The different norms discussed in lead to very different locking POD modes. Figures 3 and 4 show constant value surfaces of pressure for the four most energetic axisymmetric and first helical modes, respectively. Both show a growth and decay similar to that observed by Arndt et al. ${ }^{2}$ in pressure fluctuation adjacent the jet. Both the third and fourth most energetic modes show a streamwise period doubling consistent with a pairing phenomenon. The two most energetic modes do not show this.

Iso-surfaces of the axial and radial velocities and pressure associated with the most energetic: $K-3 \mathrm{~d}$ mode are shown in figure 5 . We see a very different character than for the pressure modes. The axial velocity, which dominates this norm, appears as long, slowly rotating streamwise structures. The radial velocity and pressure have, as they must for $n=2$, a two-fold symmetry in $\theta$ like the $u$ components, but are
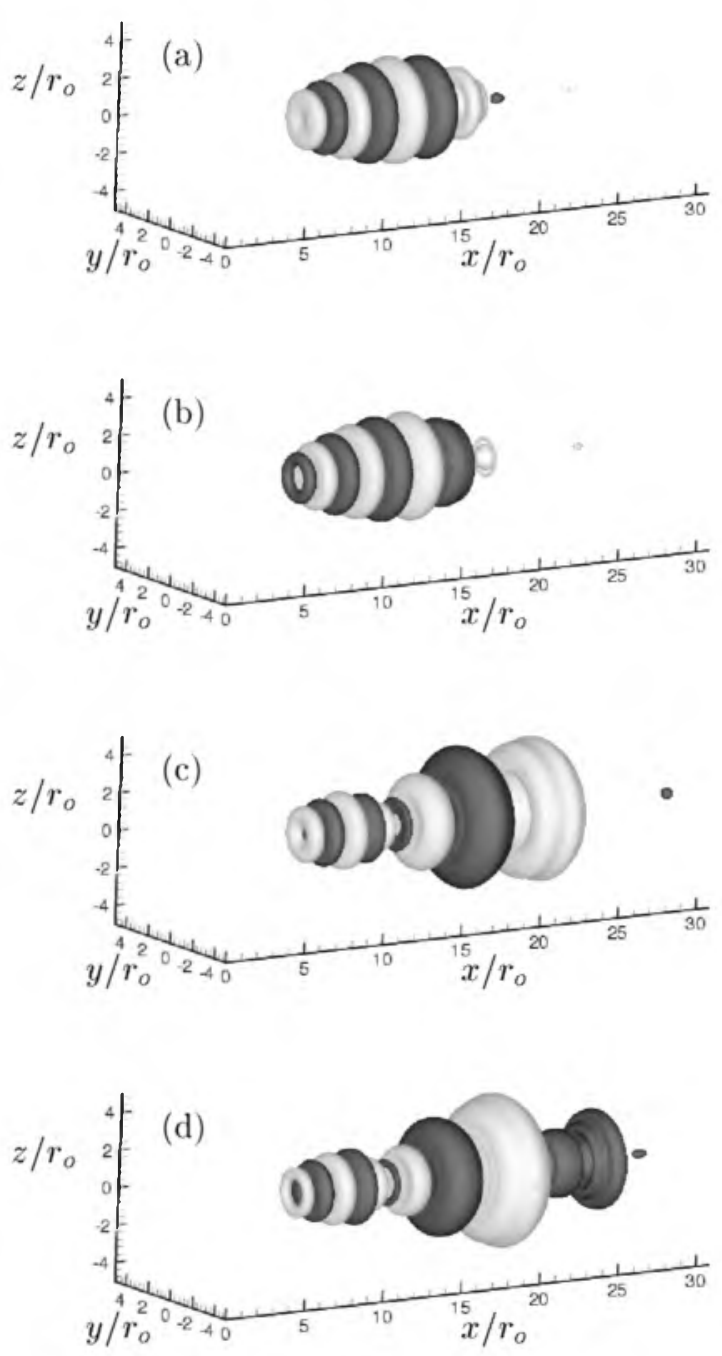

Fig. 3 Most energetic axisymmetric pressure POD modes visualized with iso-surfaces of pressure.

discontinuous in the streamwise direction.

\section{Extension to the acoustic far field}

To investigate the relation between POD modes and acoustic radiation, it is necessary to first extend the solution into the acoustic far field. The acoustic approximation is appropriate for the present sound in- 

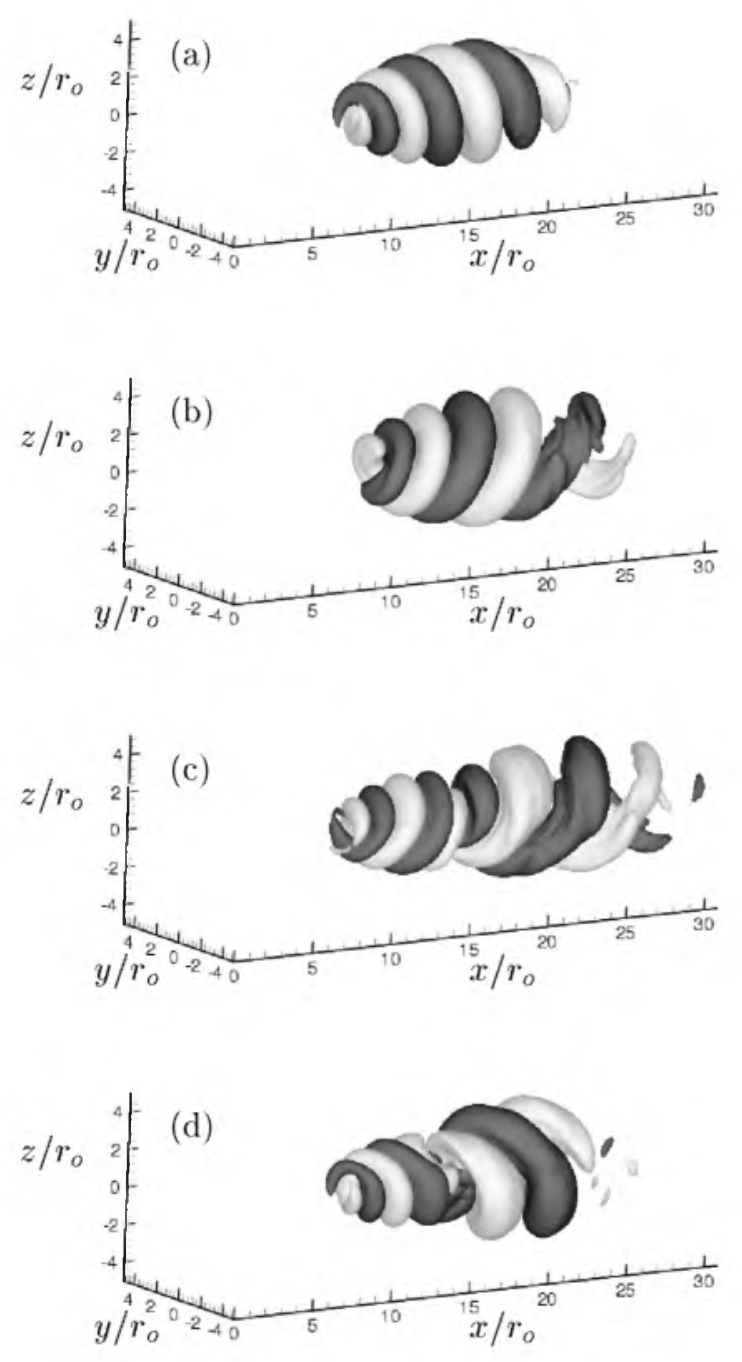

Fig. 4 Most energetic first helical $(n=1)$ pressure POD modes visualized with iso-surfaces of pressure.

tensity and the propagation distance of interest. so we assume the fluctuations are governed by the classical wave equation. Data from the flow simulation on a surface bounding the jet are used to extend to the farfield. A practical difficulty is that the truncation of the jet in the streamwise direction occurs in a region of the flow where fluctuations are nonlinear and vortical. However. Freund ${ }^{9}$ found that data on a cylinder at $r / r_{o}=8$ and ignoring the contributions from the ends produced reasonable results at angles not to shallow to the jet axis. No attempts to correct ${ }^{17}$ for the missing ends was made. It has been found that the predictions for the acoustic far field are reliable at angles greater than about 20 degrees from the jet axis.

Here we solved the wave equation in the time domain using a finite-difference method in $x$ and $r$ and a Fourier expansion in $\theta$. Sixth-order compact finite-difference and fourth-order Runge-Kutta time
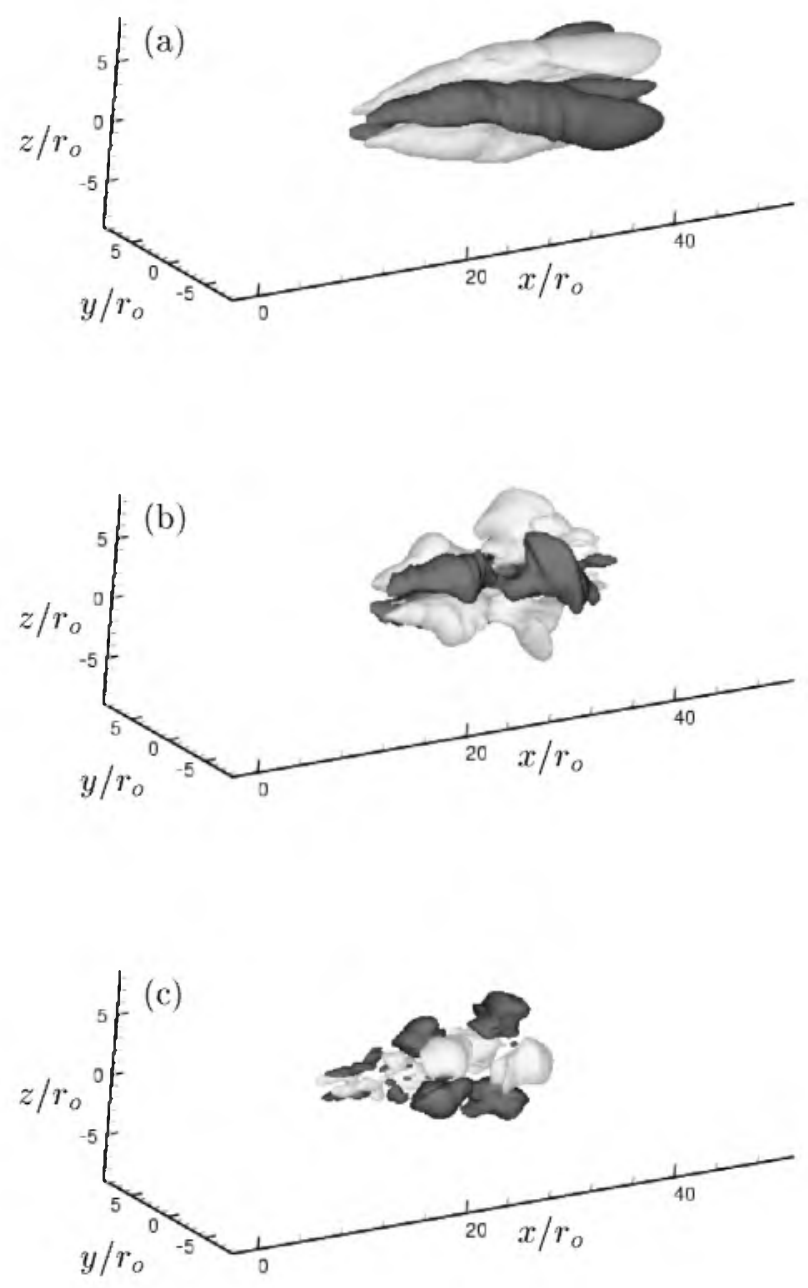

Fig. 5 Most energetic $K$-3d modes: (a) $v_{x}$; (b) $v_{r}$; and (c) $p$.

advancement were used. A Dirichlet boundary condition for the pressure was imposed along $r / r_{0}=$ 8 , and approximately nonreflecting boundary conditions $^{18}$ were used to absorb outgoing disturbances at $r=80 r_{o}$ (131 points) and $x=-20 r_{o}$ (left boundary) and $x=80 r_{o}$ (right boundary). The mesh had $151 \times 131$ point in $x$ and $r$, respectively. Because the field is linear, the azimuthal modes evolve independently and only the first 10 have so far been evaluated.

Because of the transient corresponding to the time required for the first wave emitted to propagate the length of the extended computational domain, the full acoustic field was unavailable for roughly the first quarter of the snapshots of the simulation time series. Thus in what follows, only the last 1750 of the snapshots (and the extensions to the far field) are considered.

Though the POD decomposition discussed above 


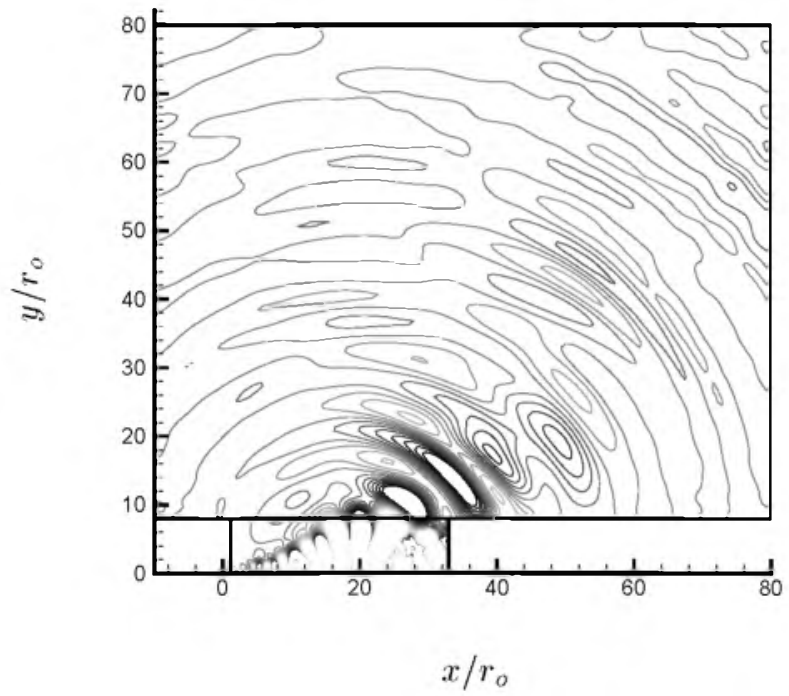

Fig. 6 Extension of the most energetic POD mode ( $P$-3d norm) for $n=1$. The real part of the complex mode is plotted with contour levels that the show acoustic field but are saturated in the near field.

works with vectors of (fluctuating) flow quantities. We only use the fluctuating pressure in the far field $(\boldsymbol{\alpha}=[0,0,0,0,1])$. Given the acoustic approximation, other far-field norms are, of course, linearly dependent on the pressure norm.

In figure 6 , we confirm that the POD modes (pressure component) computed using the far-field method described here extends smoothly between the near and far fields. This is of course necessary result if the instantaneous pressure field extends smoothly from near to far field, but it nevertheless confirms the latter and shows the extension of the view of the first azimuthal most energetic POD mode shown above in figure figure 3 .

\section{Reconstruction of flow-field statistics}

In this section we examine the extent to which a portion of the POD modes capture statistics of the flow relevant to both the near-field evolution (turbulence. kinetic energy and pressure fluctuations) and the far field sound pressure level. We examine these for several of the different norms defined in section.

For each norm, the extent to which a subset of the modes captures, on average, the given norm is the partial sum of the eigenvalues. The first.$J$ modes capture

$$
\frac{\sum_{j=1}^{J} \lambda^{(j)}}{\sum_{j=1}^{N} \lambda^{(j)}}
$$

of the total energy. However, this is not true for statistics, such as the turbulence kinetic energy at a point in the flow, or the sound pressure level at a point in the far field. The reconstruction of these quantities is easily found by simply summing the appropriate components of the eigenfunctions. For example, the (local) turbulence kinetic energy in the first $M$ modes is, under appropriate normalization of the POD modes,

$$
\begin{aligned}
q^{2}(\mathbf{x})=\frac{1}{N} \sum_{j=1}^{J} \sum_{m=-M}^{M} & \left(\varphi_{j\left(v_{x}\right)}^{m}(\mathbf{x}) \varphi_{j\left(v_{x}\right)}^{m}(\mathbf{x})\right. \\
& +\varphi_{j\left(v_{r}\right)}^{m}(\mathbf{x}) \varphi_{j\left(v_{r}\right)}^{m}(\mathbf{x}) \\
& \left.+\varphi_{j\left(v_{\theta}\right)}^{m}(\mathbf{x}) \varphi_{j\left(v_{\theta}\right)}^{m}(\mathbf{x})\right),
\end{aligned}
$$

where the second subscript on $\boldsymbol{\varphi}_{(*)}$ indicates the component of the vector mode (one of $v_{x}, v_{r}, v_{\theta}, a$, or $p$ ). Similarly, amplitude of the pressure fluctuation at any point is

$$
\overline{p^{\prime} p^{\prime}}(\mathbf{x})=\frac{1}{N} \sum_{j=1}^{J} \sum_{m=-M}^{M}\left(\varphi_{j(p)}^{m}(\mathbf{x}) \varphi_{j(p)}^{m}(\mathbf{x})\right) .
$$

If all the modes are summed, $M=M_{\theta}$ and $J=N$, we recover the total quantity, an average over all $\theta$ and all snapshots. In what follows, we have restricted our attention to sums over the first 10 azimuthal modes, $M=N_{\theta}$. When all the POD modes were used with just the 10 azimuthal modes, it was found that the statistics were essentially indistinguishable from the full statistics found by simple averaging all the original simulation data. Therefore in what follows we take $M=M_{\theta}$ and varying numbers for $J$.

It is expected that a particular norm will efficiently reproduce (with small $J$ ) the quantities on which it is based. However, it is of primary interest to investigate the efficiency with which it can reproduce other quantities. For example, how well is the far-field sound pressure level reconstructed based on the nearfield turbulence kinetic energy norm $(K-3 \mathrm{~d})$, or how well is the near-field kinetic energy (point-wise) reconstructed based on the pressure integrated over a large sphere in the far-field $(p-s p)$ ?

\section{Near-field reconstruction}

Figure 7 shows how well the turbulence kinetic energy is recovered to answer the first question. We examine the energy as a function of $r$ at two streamwise positions in the flow: $x=8 r_{o}$ and $x=16 r_{o}$. The first is representative of the shear layer fluctuations prior to the close of the potential core; the second is close to the location where the kinetic energy reaches a maximum and is near the position where the potential core closes. Shown in figure 7 are reconstructions with $J=10,50,100,500$ and all 17500 modes.

Near $x=16 r_{o}$ (figure $7 \mathrm{~b}$ ), the $K$-3d norm captures the point-wise turbulence kinetic energy most efficiently, as one might expect. But nearly 500 modes (table 1). The nine largest eigenvalues for each azimuthal mode are needed before the shape of the curve is indistinguishable from the full statistics correct. Somewhat more surprisingly, the (table 1). 


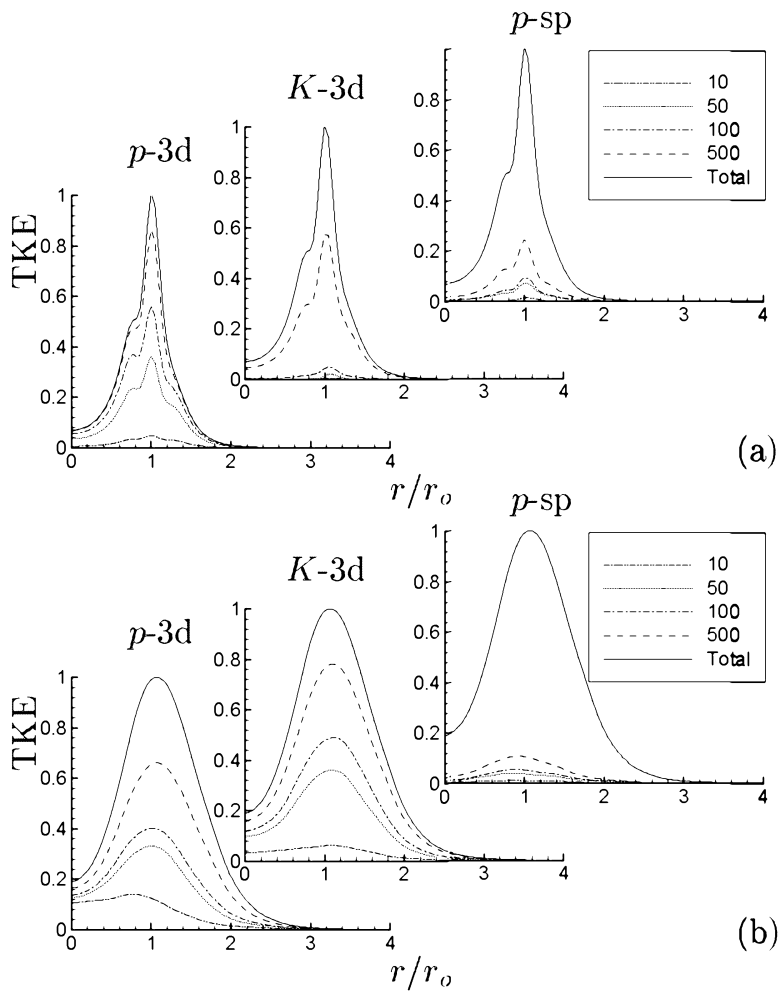

Fig. 7 Reconstruction of turbulence kinetic energy (TKE) at (a) $x=8 r_{o}$ and (b) $x=16 r_{o}$.

The nine largest eigenvalues for each azimuthal mode are shown $p-3 \mathrm{~d}$ norm (dashed lines) also captures the nearly as effectively as the $K-3 \mathrm{~d}$ norm. The $p$-sp norm is inefficient, but this is not unexpected since most energy in the near field does not directly contribute to the acoustic field. This is discussed further in the next section.

At the position further upstream (figure 7 a), we sce that the $p-3 \mathrm{~d}$ modes actually capture the pointwise turbulence kinetic energy more efficiently than the $K-3 \mathrm{~d}$ modes do (by a very considerable margin). One would expect more efficient reconstruction downstrcam where the fluctuations (both turbulence energy and pressure) are larger (and therefore contribute more to the norm). But for the pressure the reconstruction is equally good at both locations, despite the fact that the pressure fluctuations are an order of magnitude smaller at $x=8 r_{o}$ than at $x=16 r_{o}$. It is interesting to further compare the reconstruction of $\overline{p^{\prime} p^{\prime}}$ as is done for the same streamwise positions in figure 8 . In both cases the $p$ - $3 \mathrm{~d}$ norm also better captures the point-wise pressure fluctuations in fewer modes. This better convergence may have to do with the fact that the most energetic $K$-3d modes appear to primarily represent large structures (with $n=2$ dominant) in the region somewhat downstream of the close of the potential core.

In gencral, it may at first seem disappointing to require 500 modes to capture only about $60 \%$ of the peak kinctic cncrgy at $x / r_{o}=16$. In many flows
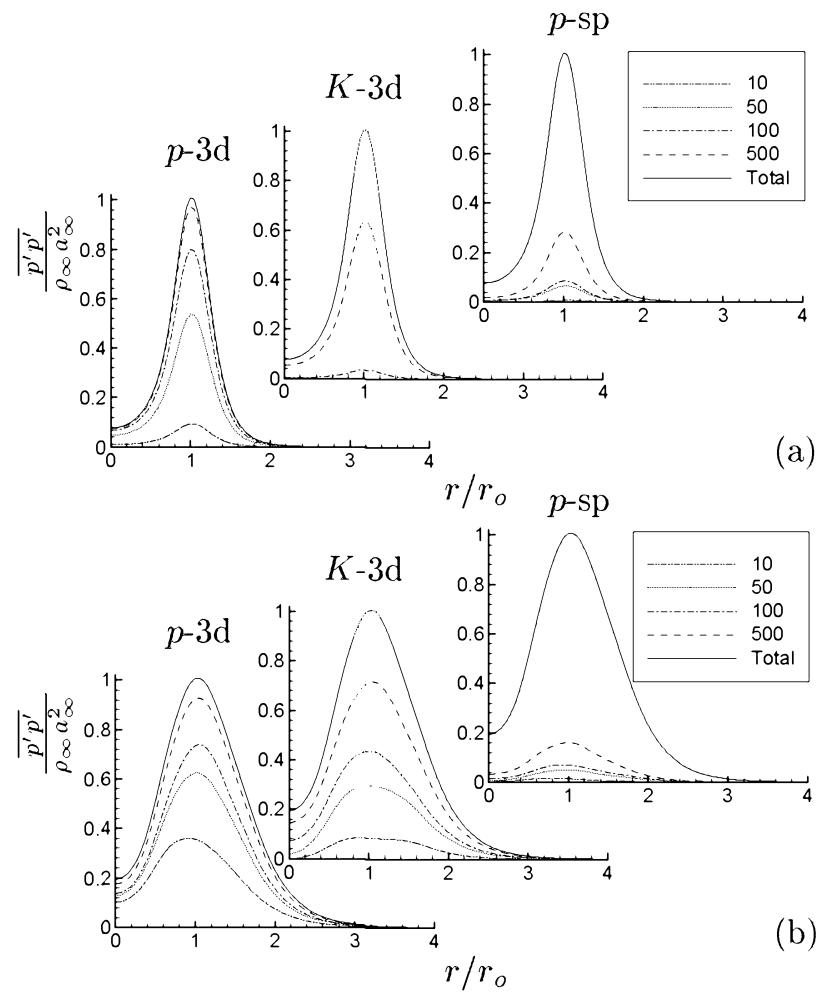

Fig. 8 Reconstruction of pressure fluctuations at (a) $x=8 r_{o}$ and (b) $x=16 r_{o}$.

one would hope for only a few modes to be dominant, to capture some relevant dynamical feature of the flow at low-order. However, when one considers that this is just $2.86 \%$ of the modal content of the data set (there were 10 azimuthal modes and 1750 snapshots, so $500 / 10 / 1750=2.86 \%$ ), it seems more impressive. Clearly further work is warranted to determine whether reduced-order models based on Galerkin projection of the modes have the ability to capture a similar portion of the energy or pressure, and to determine whether the lowest-order modes reveal something interesting about the dynamics of the turbulence.

\section{Far-field reconstruction}

We turn now to the reconstruction of the acoustic field by the POD modes. As in the last section, we sum over the first 10 azimuthal modes and various numbers of POD modes, $J$. Despite the fact that we used 10 modes, modes $n=0$ and $n=1$ are dominant in the far-field, with virtually no contribution from the higher azimuthal modes. We consider the data on a large spherical shell at $R=\sqrt{x^{2}+r^{2}}=60 r_{o}$, the same area over which the $p$-sp norm was defined. Plotted in figure 9 is $\overline{p^{\prime} p^{\prime}}$ versus directivity angle $\psi$ measured from the downstream axis, for reconstructions with $J=10,50,100$, and 500 modes. The peak radiation is at roughly $\psi \approx 30^{\circ}$ for the total and most of the reconstructions. The dotted line shows reconstruction based on the $p$-sp norm. It is not surprising that only a few modes, so-defincd, capture the radi- 

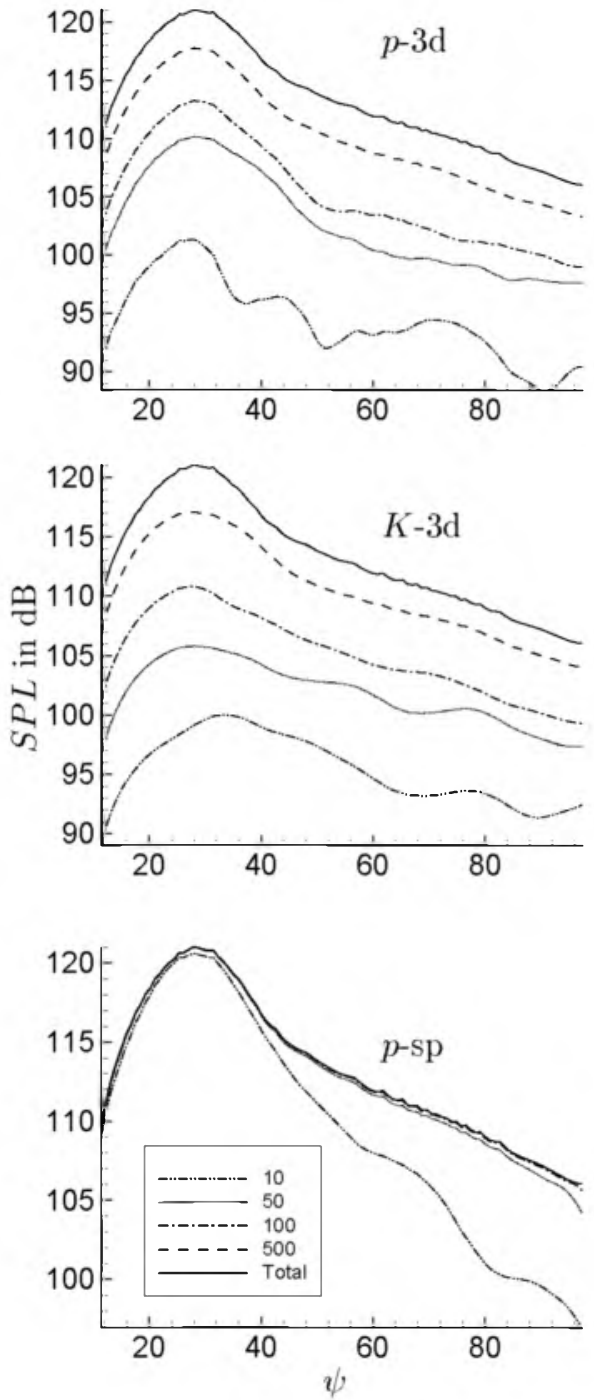

Fig. 9 Reconstruction of the far-field sound pressure level.

ation. It was already remarked in section that area based norms converge much more rapidly than volume based norms. Moreover, the far-field fluctuations are are made up of relatively lower frequency contributions than the near field.

We see in figure 9 that for the near-field norms, $K$ $3 \mathrm{~d}$ and $p-3 \mathrm{~d}$, we obtain relatively poorer convergence of the POD partial sums (the $p-3 \mathrm{~d}$ norm is somewhat better with fewer modes as before). Because the field is dominated by the first two azimuthal modes, the compression factor less impressive than for the near field. For example, with 500 modes here, we are really only compressing by $500 / 2 / 1750=14 \%$.

It is interesting to inquire about the inverse process: given the $p$-sp modes, how well are the near-field quantities reconstructed? The near-field reconstructions were already shown in figures 7 and 8 , and it was remarked in the previous section that the $p$-sp modes had relatively poor convergence compared to

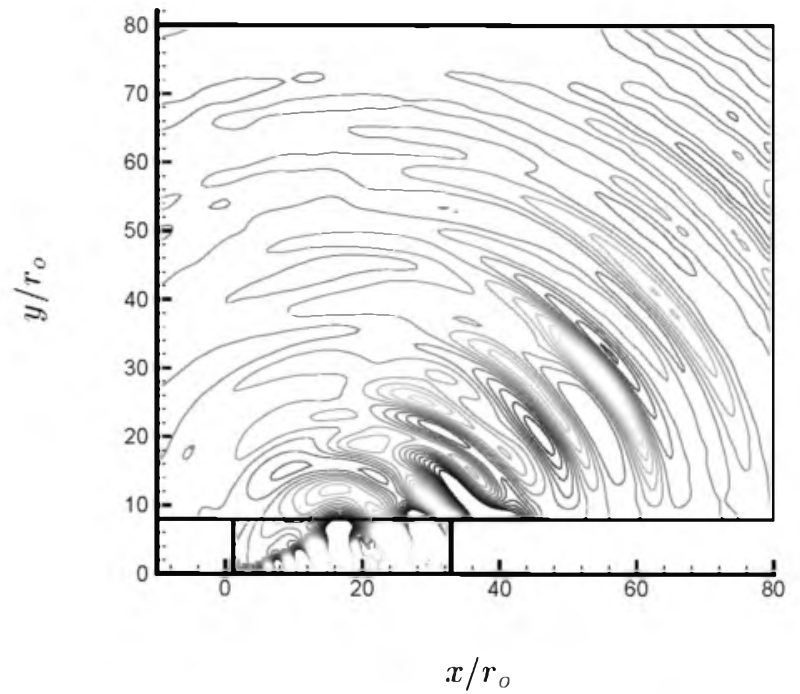

Fig. 10 Extension of the most energetic POD mode ( $p$-sp norm) for $n=1$. The real part of the complex mode is plotted with contour levels that the show acoustic field but are saturated in the near field.

the modes based on near-field norms. This is not surprising, however, when one considers that a very small fraction of the near-field energy is radiated as sound.

Finally, it is of interest to examine the near-field structure of the dominant modes contributing to the far-field norm, as this may reveal something about the mechanism of sound generation. In figure 10 we show the most energetic mode, which corresponds to azimuthal mode $m=1$. The pressure contours again show a fairly regular wave packet, not dissimilar to that produced by the dominant $p$-sp mode (figure 6 ). However, the latter mode has a very regular structure throughout (figure 4) while the mode educed from the far-field norm has an abrupt change in structure in a region localized to the end of the potential core. It is known (e.g. Freund ${ }^{9,19}$ ) that rapid disruption of the wave packet structure, as might occur near the end of the potential core, (:an radiate noise efficiently. Further work is needed to see whether this structure is well-correlated with the radiating portion of the Lighthill source. If so, then such POD modes may be a valuable component of a reduced-order model for the sound radiated by the large-scale turbulence in the jet.

\section{Summary}

We have presented POD modes computed for various energy norms for a jet, showing qualitative agreement with experimentally derived POD expansions based on streamwise velocity. We find that norms that involve integration over three dimensions lead to POD expansions that convergence considerably more slowly than those whose norm is defined as an integral in two 
dimensions (as is most often done in experiments due to measurement difficulties).

POD modes computed for a pressure volume come in pairs and have a clear wave-packet structure. Other norms give very different distributions of energy amongst the modes and very different looking eigenfunctions.

To converge to $50 \%$ of the turbulence kinetic energy (globally) requires 100 modes from a turbulence kinetic energy norm, $0.57 \%$ of the total modes used to represent the full flow statistics. The pressure norm is nearly as efficient (better in some places) at representing the energy. However, obtaining a good representation of the sound field requires virtually all the POD modes from either TKE or pressure based norms. Of course, one may define the norm as an integration over the far field and obtain rapid convergence to the radiated sound. Such a norm does not offer an efficient compression of the near-field data, but the modes that it does illuminate appear to have an interesting wavepacket appearance and may be dynamically relevant to the sound radiated by large structures in the flow.

\section{Acknowledgments}

JBF gratefully acknowledges the support of NASA. Both authors acknowledge fruitful interactions with Dr. Peter Blossey whose initial computations of pressure POD modes from this database inspired the present effort.

\section{References}

${ }^{1}$ Holmes, P., Lumley, J. L., and Berkooz, G.. Turbulence, Coherent S'truclures, Dynamical Systems and Symmetry, Camtoridge University Press, Cambridge, 1996.

${ }^{2}$ Arndt, R. E. A., Long, D. F., and Glauser. M. N., "The proper orthogonal decomposition of pressure fluctuations surrounding a turtulent jet," .J. Fluid Mech., Vol. 340, 1997, pp. 1$3: 3$.

"Gordeyev, S. V. and Thomas, F. O., "Coherent structure in the turbulent planar jet Part 1. Extraction of proper orthogonal decomposition eigenmodes and their self-similarity." J. Fluid Mech., Vol. 414, 2000, pp. 145-194.

${ }^{4}$ Rowley, C., Colonium, T., and Murray, R., "Lynamical Models for Control of Cavity Oscillations," $7^{\text {th }}$ ALAL/CE.LS Aeroacoustics Meeting, Maastrich, 'The Netherlands. 28 - 30 May 2001, ALA Paper 2001-212f, 2001.

"Moin. P. and Moser, R. D., "Characteristic-eddy decomposition of turbulence in a channel," J. Fluid Mech. V'ol. 200. 1989 , pp. $471-509$.

${ }^{6}$ Jung. D.. An investigation of the Reynolds-number dependence of the axisymmetric jel mixing layer using a 138 hot-wire probe and the POD. Ph.D. thesis, State University of New York at Buffalo. September 2001.

'Citriniti. J. H. and George, W. K., "Reconstruction of the glotal velocity field in the axisymmetric mixing layer utilizing the proper orthogonal decomposition," J. Fluid Mech.. Vol. 418. 2000 , pp. $137-166$.

'Jung. D.. Gamard. S., George, W. K., and Woodward. S. H.. "Downstream evolution of themost energetic: POL) modes in the mixing layer of a high keynolds number axisymmetric: jet," Proceedings of the IUTAM Symposium on Turbulent Mixing and Combustion. Queens University at Kingston. Canada. June $3-6.2001$
${ }^{9}$ Freund, J. B., "Noise sources in a low-Revnolds-number turbulent jet at Mach 0.9." J. Fluid Mech., Vol. 438, 2001. pp. 277-305.

${ }^{10}$ Stromberg, J. L., McLaughlin, D. K.. and Troutt, T. R., "Flow field and acoustic properties of a Mach number 0.9 jet at a low Reynolds number." J. of Sound and Vib.. Vol. 72, No. 2, 1980, pp. 159-17fi.

${ }^{11}$ Hussein, H. J., Capp, S. P', and George, W. K., "Velocity measurements in a high-Reynolds-number, momentumconserving, axisymmetric: turbulent jet," J. Fluid Mech., Vol. 258, 1994, pp. :31-75.

${ }^{12}$ Panchapakesan, N. K. and Lumley, J. L., "Turbulence measurements in axisymmetric jots of air and helium. Part. 1. Air jets," J. Fluid Mech., Vol. 246, 1993, pp. 197223.

${ }^{13}$ Rowley, C. W., Modeling, simulation and control of cavity flow oscillations, Ph.D. thesis, California Institute: of Technology, 2002.

${ }^{14}$ Sirovich, L., "Chaotic: dynamics of coherent structures. Parts 1-111," Quarterly of Applied Math., Vol. XLV, Vo. 3, 1987 , pp. $5 f 1-582$.

${ }^{15}$ Gamard, S., George, W. K., Juns, D., and Woodward, S. "Application of a sslice" POD to the far field of an axisummetric turbulent jet," personal communication, 2001.

${ }^{16}$ Fuchs, H. V. and Michalke. A., "On turbulence and noise: of an axisvmmetric shear flow:" J. Fluid Mech.. V'ol. 70, 1975, pp. $179-205$

${ }^{17}$ Freund, J. B., " $\Lambda$ propessed inflow/outflow boundary condition for direct computation of aerodynamic sound." $\Lambda I \Lambda \Lambda \mathrm{J}$. Vol. 35, No. 4, 1997, pp. 740742.

${ }^{18}$ 'Thompson, K. W., "Time-dependent boundary" conditions for hypertolic: systems," J. Comp. Phys.. Vol. fis. 1987, pp. 124.

${ }^{19}$ Freund, J. B., "Acoustic sources in a turtulent jet: a direct numerical simulation study;" AIAA Paper 99-18.58, 1999. 